\title{
Zukünftige Antiallergika wirken breiter und sicherer
}

$\mathrm{R}$ echtzeitig zum neuen Jahrtausend wurden auf der DDG-Tagung im Mai 2001 in Berlin neue Antiallergika vorgestellt. Beispiele hierfür sind Desloratadin und Levocetirizin. Sie lösen die bisherigen Antihistaminika der 2. Generation aufgrund ihrer Vorteile in Wirkung und Sicherheit ab. Das gilt insbesondere für Desloratadin. Während bei Levocetirizin das unwirksame Isomer des Cetirizin abgetrennt wurde, handelt es sich bei Desloratadin um den wirksamen Metaboliten des Loratadin. Das heißt, es muss nicht mehr in der Leber metabolisiert werden. Das hat, wie R. Hein von der Klinik und Poliklinik für Allergologie und Dermatologie am Biederstein der TU München betont, erhebliche Vorteile in puncto Wirkung und Sicherheit. So tritt bei Desloratadin nicht nur die Wirkung schneller ein, sondern es fallen auch einige Nebenwirkungen fort. Ein Beispiel hierfür sind die Wechselwirkungen mit anderen Medikamenten oder Nahrungsmitteln. Diese können, wie uns die Vergangenheit bei Terfenadin lehrte, zu bösen Folgen führen, z.B. zu Herzrhythmusstörungen mit tödlichem Ausgang. Solche Wechselwirkungen sind bei Desloratadin auszuschließen.

\section{Juckreiz senkt Lebensqualität}

Die bisherigen Antihistaminika weisen in der Behandlung der Symptome der allergischen Rhinitis und der Urtikaria Lücken auf. Bisher konnte man weder die Obstruktion bei der Rhinitis wirkungsvoll behandeln noch den Juckreiz bei Hauterkrankungen unterdrücken. Mit Desloratadin werden diese Wirkungslücken geschlossen. So wurden auf der DDG-Tagung mehrere Studien mit Desloratadin mit ähnlich guten Ergebnissen vorgestellt, zum einen Plazebokontrollierte Doppelblindstudien, zum anderen eine multizentrische, aber offene Phase-III-Studie. An der offenen PhaseIII-Studie, die federführend von der Klinik und Poliklinik für Dermatologie und Allergologie am Biederstein der Technischen Universität München durchgeführt wurde, nahmen 150 Patienten mit chronischer idiopathischer Urtikaria teil, davon 55 Männer und 95 Frauen. Alle Patienten erhielten die Normaldosis von 5 mg Desloratadin/Tag. 51\% der Patienten mit schwerer Urtikaria waren nach vier Wochen Behandlungszeit vollkommen beschwerdefrei. Bei den anderen sah Hein eine erhebliche Verbesserung der Symptome. Etwa 50\% der Patienten konnte bereits nach einer Woche ohne den quälenden Juckreiz durchschlafen,
70\% nach vier Wochen. Die Zahl der Quaddeln reduzierte sich innerhalb der ersten Woche deutlich. Das bedeutete für die Patienten eine erhebliche Verbesserung ihrer Lebensqualität. Patienten, die in der Studie schlechter auf die Therapie ansprachen, wurden nach Studienende mit der doppelten Dosis Desloratadin wirkungsvoll ohne zusätzliches Risiko behandelt. Das geringe Nebenwirkungsprofil, betonte Hein, ließe im Gegensatz zu den Antihistaminika der 2. Generation eine höhere Dosierung zu.

Nach Informationen von Essex Pharma

\section{SIT: Fortschritt durch depigmentierte Allergenextrakte}

$M$ it der Entwicklung depigmentierter Allergen-Extrakte hat sich eine neue Generation von Allergenen für die spezifische Immuntherapie (SIT) angekündigt. Sie versprechen eine hochspezifische Immunantwort verbunden mit sehr guter Verträglichkeit.

Im Unterschied zu bisher verfügbaren Allergen-Extrakten enthält der Herstellungsprozess von Depigoid ${ }^{\circledR}$ einen zusätzlichen Reinigungsschritt, der störende Pigmente weitgehend entfernt. Den Patienten kommt dies durch deutlich weniger unerwünschte Reaktionen zugute. Denn die in nativen AllergenExtrakten enthaltenen Flavonoide, Melanoidine und Tannine führen in hohem Maße zu einer unspezifischen Aktivierung des Komplementsystems.

Der Vorteil dieses innovativen Herstellungsverfahrens schlägt sich in einer hohen Spezifität der Immunantwort nieder, erklärte E. Fernandez-Caldas (Madrid). Depigoid ${ }^{\circledR}$ zeichne sich durch eine signifikante Reduktion der IgE-Bindungskapazität im Vergleich zu nicht depigmentierten Allergoiden aus. Die gewünschte immunologische Aktivität der Allergene werde davon aber nicht beeinträchtigt, wie die unveränderte IgG-Bindungskapazität und die Stimulierung der T-Zellen in In-vitroTests beweisen würden.
Auch die klinische Wirksamkeit der Immuntherapie mit Depigoid ${ }^{\circledR}$ sei in zahlreichen Studien gut dokumentiert.

\section{Vereinfachtes \\ Behandlungsschema}

Der hohe Polymerisationsgrad von Depigoid $^{\circledR}$ ermöglicht die Anwendung einer hohen Allergenkonzentration pro Dosis. Damit verkürzt sich die Zeit bis zum Eintreten der Immunantwort, ohne die Verträglichkeit negativ zu beeinflussen. Dass die SIT insgesamt weniger Injektionen als sonst üblich erfordert, dürfte für die Patienten ein ebenso wichtiges Argument sein wie das einfache Behandlungsschema. Die Erhaltungsdosis wird nämlich bereits nach drei Wochen erreicht, die weiteren Behandlungen erfolgen im Abstand von jeweils vier Wochen.

Depigoid $^{\circledR}$ steht seit 1 . September 2001 für die wichtigsten Gräser-, Baum- und Kräuterpollen sowie MilbenAllergene zur Verfügung. Katzen- und Schimmelpilz-Allergene werden im nächsten Jahr hinzukommen. $J N$

Satellitensymposium anlässlich der 4. Jahrestagung der Gesellschaft für Pädiatrische Allergologie und Umweltmedizin, München 2001. Veranstaltet von Dr. Beckmann 